\title{
Revealing brain pathologies with multimodal visible light optical coherence microscopy and fluorescence imaging
}

\author{
Antonia Lichtenegger \\ Johanna Gesperger \\ Barbara Kiesel \\ Martina Muck \\ Pablo Eugui \\ Danielle J. Harper \\ Matthias Salas \\ Marco Augustin \\ Conrad W. Merkle \\ Christoph K. Hitzenberger \\ Georg Widhalm \\ Adelheid Woehrer \\ Bernhard Baumann
}




\title{
Revealing brain pathologies with multimodal visible light optical coherence microscopy and fluorescence imaging
}

\author{
Antonia Lichtenegger, ${ }^{\mathrm{a}, \star}$ Johanna Gesperger, ${ }^{\mathrm{a}, \mathrm{b}}$ Barbara Kiesel, ${ }^{\mathrm{c}}$ Martina Muck, ${ }^{\mathrm{a}, \mathrm{b}}$ Pablo Eugui, ${ }^{\mathrm{a}}$ \\ Danielle J. Harper, ${ }^{a}$ Matthias Salas, ${ }^{a}$ Marco Augustin, ${ }^{a}$ Conrad W. Merkle, ${ }^{a}$ Christoph K. Hitzenberger, \\ Georg Widhalm, ${ }^{c}$ Adelheid Woehrer, ${ }^{b}$ and Bernhard Baumann ${ }^{\mathrm{a}}$ \\ a Medical University of Vienna, Center for Medical Physics and Biomedical Engineering, Vienna, Austria \\ ${ }^{\mathrm{b}}$ General Hospital and Medical University of Vienna, Institute of Neurology, Vienna, Austria \\ ${ }^{\circ}$ General Hospital and Medical University of Vienna, Univ. Klinik Neurochirurgie, Vienna, Austria
}

\begin{abstract}
We present a multimodal visible light optical coherence microscopy (OCM) and fluorescence imaging (FI) setup. Specification and phantom measurements were performed to characterize the system. Two applications in neuroimaging were investigated. First, curcumin-stained brain slices of a mouse model of Alzheimer's disease were examined. Amyloid-beta plaques were identified based on the fluorescence of curcumin, and coregistered morphological images of the brain tissue were provided by the OCM channel. Second, human brain tumor biopsies retrieved intraoperatively were imaged prior to conventional neuropathologic work-up. OCM revealed the three-dimensional structure of the brain parenchyma, and FI added the tumor tissue-specific contrast. Attenuation coefficients computed from the OCM data and the florescence intensity values were analyzed and showed a statistically significant difference for 5-aminolevulinic acid (5-ALA)-positive and -negative brain tissues. OCM findings correlated well with malignant hot spots within brain tumor biopsies upon histopathology. The combination of OCM and FI seems to be a promising optical imaging modality providing complementary contrast for applications in the field of neuroimaging. ๑ The Authors. Published by SPIE under a Creative Commons Attribution 4.0 Unported License. Distribution or reproduction of this work in whole or in part requires full attribution of the original publication, including its DOI. [DOI: 10.1117/1.JBO.24.6.066010]
\end{abstract}

Keywords: optical coherence tomography; supercontinuum laser; Alzheimer's disease; glioma; 5-aminolevulinic acid; protoporphyrin IX. Paper 190116R received Apr. 12, 2019; accepted for publication Jun. 7, 2019; published online Jun. 25, 2019.

\section{Introduction}

Optical coherence tomography (OCT) is an optical imaging technique, which was introduced in the early 1990's. OCT has since become one of the most important imaging and diagnosis tools in ophthalmology. ${ }^{1}$ The use of OCT or optical coherence microscopy (OCM) also expanded into a wide range of application fields such as neuro-, skin, and endoscopic imaging. ${ }^{2-4}$ In neuroimaging, OCT has been utilized to investigate diseases such as Alzheimer's disease (AD), ${ }^{5-7}$ Parkinson's disease, ${ }^{8,9}$ and a variety of brain tumors. ${ }^{10-12}$ However, in comparison to histology, OCT often lacks tissue-specific contrast of anatomical structures since conventional OCT image contrast is mainly based on light scattering. Current research in the OCT community has therefore also focused on validating and/or combining OCT with other established imaging modalities. One promising possibility is to combine OCT and fluorescence imaging (FI). ${ }^{13}$

FI provides tissue specificity based on biochemical or metabolic contrast through either autofluorescence or exogenous fluorescent dyes. ${ }^{14}$ A combination of OCT and FI may enable the assessment of tissue morphology by OCM imaging with complementary biochemical tissue information retrieved by FI. Multimodal OCT and FI approaches have been implemented in different ways by research groups over the past years. ${ }^{15-35}$ However, most presented OCM/fluorescence systems thus far have been based on rather complex optical layouts, which were

*Address all correspondence to Antonia Lichtenegger, E-mail: antonia .lichtenegger@meduniwien.ac.at designed to only work for one specific fluorescent dye and often relied on two separate light sources and paths, one for OCM and one for FI. Also, most of the OCT subsystems were operated in the near-infrared wavelength region and were designed for imaging the retina. Recently, using supercontinuum lasers working in the visible light spectrum, submicrometer axial resolutions for imaging the eye ${ }^{34,36-38}$ cells,${ }^{39}$ and brain tissue ${ }^{5-7}$ were achieved. $^{40}$

$\mathrm{AD}$ is characterized by the degeneration of neurons, the formation of extracellular plaques composed of amyloid-beta protein, and the accumulation of intracellular neurofibrillary tangles composed of tau protein. ${ }^{41}$ In our recent work, we investigated neuritic amyloid-beta plaques in $\mathrm{AD}$ brain tissue using a high-resolution visible light OCM setup. ${ }^{6,7}$ In histology, which is the gold standard for confirming these structures in neuropathology, amyloid-beta plaques can be identified using various stains, such as Congo red or immunohistochemical staining against amyloid-beta. ${ }^{42,43}$ Another possibility to visualize amyloid-beta plaques is the use of fluorescent dyes, such as thioflavin-S or curcumin. ${ }^{44-46}$ Curcumin is a yellow pigment, which is extracted from the rhizome of the plant Curcuma longa and has been used to label amyloid-beta deposits ex-vivo. ${ }^{47}$

Optical imaging plays a crucial role during dissections of brain tumors. In state-of-the-art neurosurgery, the surgeon utilizes an intraoperative microscope to navigate through the procedure and to localize malignant tissue areas to be resected. ${ }^{48}$ The most common and most aggressive primary brain tumor is the glioblastoma multiforme, which belongs to a group of tumors called gliomas. Even with the best possible treatment, 
in most cases median survival does not exceed 15 months. Surgery followed by radiotherapy and concomitant temozolomide chemotherapy is the most important current treatment approach. ${ }^{49}$ To enable maximal safe resections, intraoperative differentiation of tumor tissue and brain parenchyma is of utmost importance. ${ }^{50}$ Recently, 5-aminolevulinic acid (5-ALA) FI has emerged as a powerful intraoperative modality capable of detecting high-grade glioma. The patient orally intakes the 5-ALA tracer prior to surgery. 5-ALA is then metabolized in the tumor cells to the fluorescent molecule protoporphyrin IX (PPIX).${ }^{51}$ During the operation, a blue light source, integrated in the neurosurgical microscope, is used to excite the fluorophore, making it easier for the surgeon to identify the malignant areas. ${ }^{52-54}$ However, FI lacks morphological information about the tissue. It was already shown that OCT is a promising technique to investigate tumorous brain tissue. ${ }^{12,55-59}$ Kut et al. ${ }^{12}$ evaluated the attenuation coefficient extracted from the OCT data to distinguish between healthy tissue and tumors at different stages. Researchers have focused on integrating OCT into surgical microscopes. ${ }^{60-62}$

In this work, we present an adaptable, multimodal visible light OCM and FI setup. Our system is based on a supercontinuum laser operating in the visible wavelength range as a single, shared light source for both OCM and FI. By using a visible spectrum and appropriate spectral filter sets, the presented setup is able to excite various fluorescence dyes. In the OCM channel, the broad spectrum provides a high axial resolution to investigate microscopic features in the tissue. We present imaging of amyloid-beta plaques, in ex-vivo brain tissue of a mouse model of $\mathrm{AD}$, using curcumin-based fluorescence contrast and visualize the three-dimensional structure of the brain tissue using the OCM channel of our multimodal setup. Finally, we investigate ex-vivo tumor biopsies with our multimodal setup. We identify the malignant regions with the FI setup and assess the morphological information of the brain parenchyma with the OCM. Finally, we evaluate the attenuation coefficients retrieved from the OCM measurements and perform a comparison to histology.

\section{Methods}

\subsection{Combined Visible Light Optical Coherence Microscopy and Fluorescence Imaging Setup}

A multimodal visible light optical coherence microscope (OCM) and FI setup was developed. The OCM subsystem was first introduced in 2017..$^{6}$ A sketch of the setup is shown in Fig. 1(a).

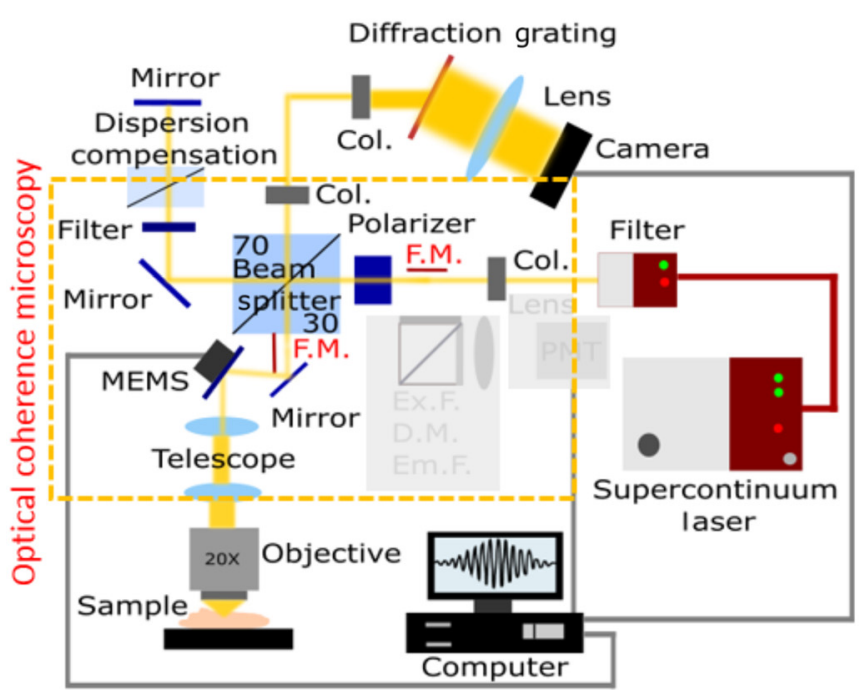

(a)

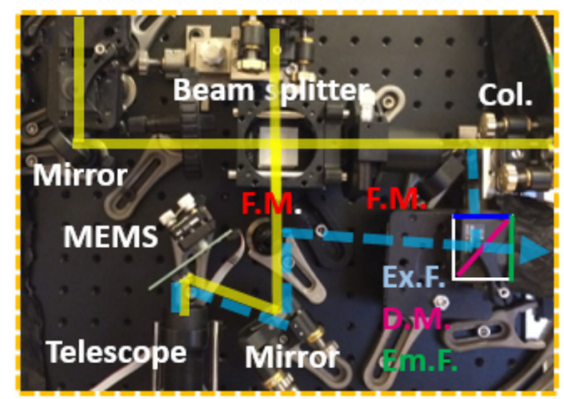

(c)

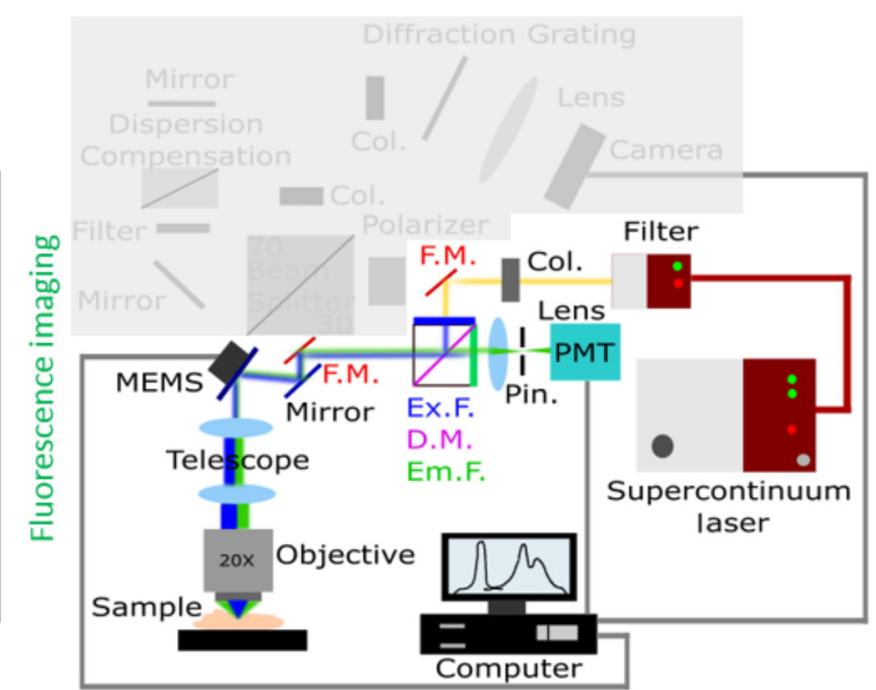

(b)

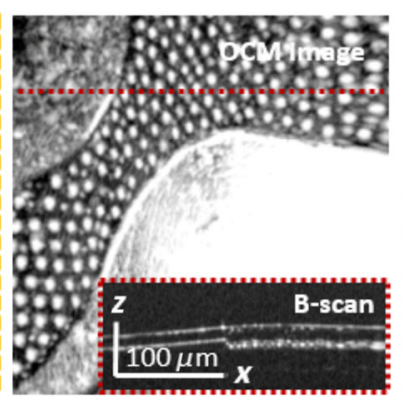

(d)

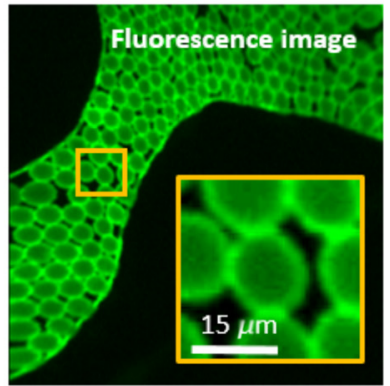

(e)

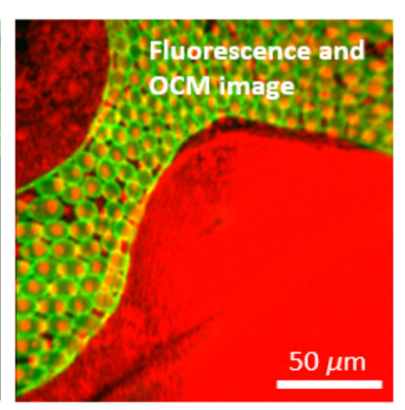

(f)

Fig. 1 The multimodal FI and visible light OCM setup. (a) Sketch of the visible light OCM subsystem. (b) The FI subsystem after flipping the mirrors into the beam (indicated as F.M. in red). Components: collimator (Col.), dichroic mirror (D.M.), emission filter (Em.F.), excitation filter (Ex.F.), flip mirror (F.M.), pinhole (Pin.), and PMT (photomultiplier tube). (c) Photograph of a part of the interferometer of the OCM, where the yellow line indicates the laser beam of the OCM part and the blue dashed line the beam of the FI part. (d)-(f) A phantom of dragon-green microbeads was imaged. (d) OCM en-face projection and a representative B-scan. (e) Fl image with a zoom-in. (f) Overlay of the OCM (red) and Fl (green) images. 
The system is based on a free-space Michelson interferometer and a custom-built spectrometer. A supercontinuum laser in combination with a variable filter box (NKT Photonics SuperK EXTREME EXU-6 and VARIA) provided a broad visible spectrum (400 to $700 \mathrm{~nm}$ ). An axial resolution of $0.88 \mu \mathrm{m}$ in brain tissue was measured assuming a refractive index of $1.36 .{ }^{63}$ A $20 \times$ magnification objective lens was utilized (Olympus, UPLFLN 20XP) leading to a measured transversal resolution of $2 \mu \mathrm{m}$ and a theoretical depth of focus of $44 \mu \mathrm{m}$. The A-scan rate of the system was $30 \mathrm{kHz}$. To switch from OCM to FI, two flipping mirrors (F.M.), see Fig. 1(b), were used to deflect the beam. A zoom-in photograph of the combined setup in Fig. 1(c) shows the part where the two mirrors can be flipped. For FI, a variable combination of excitation filter (Ex.F.), dichroic mirror (D.M.), and emission filter (Em.F.) can be chosen and inserted in a filter cube (Olympus U-MF2) and may therefore enable flexible imaging of a variety of fluorophores. The light beam first passed through an excitation filter (Thorlabs MF 434-17, central wavelength $=434 \mathrm{~nm}$, bandwidth $=17 \mathrm{~nm}$ ) and was reflected by a dichroic filter (Thorlabs MD 434) and scanned across the sample. The beam in the sample arm was raster scanned using a microelectromechanical mirror scanner (MEMS, Mirrorcle Technologies, Inc.). The line scan camera (Basler spL8192$70 \mathrm{~km}$ ) in the spectrometer, the MEMS and the PMT were synchronized using a custom-made LabView [Version 15.0 (64-bit)] program. The photons emitted by sample fluorescence passed through an emission filter (Curcumin: Thorlabs MF 53043 , central wavelength $=530 \mathrm{~nm}$, bandwidth $=43 \mathrm{~nm}$; 5-ALA: MF 630-69, central wavelength $=630 \mathrm{~nm}$, bandwidth $=$ $69 \mathrm{~nm}$ ). The fluorescent signal was focused by a lens onto a photomultiplier tube (Thorlabs, PMMT02, PMT), whose voltage output was digitized using a data acquisition card (National Instruments PCIe-6321). A pinhole with a diameter of $700 \mu \mathrm{m}$ was placed in the focal plane before the PMT to reduce of outof-focus light. For OCM imaging, the power at the sample was measured to be $0.8 \mathrm{~mW}$ and for FI $0.2 \mathrm{~mW}$.

\subsection{Tissue Preparation}

\subsubsection{Phantom imaging}

To validate the setup, three fluorescence phantoms were imaged. For the first phantom, mounting medium (Aqua-Poly/Mount, Polysciences) was mixed (concentration 10\%) with a curcumin powder (Sigma-Aldrich). This mixture was stuck onto paper and a region including both paper and curcumin was imaged. As a control case, only mounting medium on paper was imaged. Second, to verify the system's 5-ALA imaging capabilities, a fluorescence reference target (Starna Scientific, Protoporphyrin IX in PMMA, concentration 20\%) was utilized (data not shown). For the third phantom dragon-green micro-beads (polystyrene-based microspheres dyed with dragon-green fluorophore) were imaged, and the results are shown in Figs. 1(d)1(e). Figure 1(d) shows the OCM en-face image averaged over $10 \mu \mathrm{m}$ in depth as well as a representative B-scan. Figure 1(e) shows the corresponding FI image including a zoom-in. Figure 1(f) shows an overlay of the OCM (red) en-face projection and the FI image (green).

\subsubsection{Curcumin-stained mouse brain tissue}

Heterozygous breeding of an $\mathrm{AD}$ mouse model, APPswe, PSEN1dE9 (APP-PS1, MMRRC stock number 34829, The
Jackson Laboratory ${ }^{64-66}$ ) was established. Animal experiments were approved by the local ethics committee and by the Austrian Federal Ministry of Education, Science and Research under protocol BMWFW-66.009/0279-WF/V/3b/2018. One mouse was sacrificed at the age of 63 weeks. The brain was carefully removed and for one hemisphere a vibratome (Vibratome Series 1000 Sectioning System, The Vibratome Company) was used to cut $100-\mu \mathrm{m}$-thick brain sections. The slices were cleared for 15 min following the SWITCH clearing protocol, ${ }^{6,67}$ stained with curcumin, and imaged using a laser scanning microscope (Confocal Microscope LSM 700 Zeiss). The other hemisphere was embedded in paraffin and $3 \mu \mathrm{m}$ thick slices were stained with curcumin and imaged by a conventional Olympus fluorescence microscope (BX51). Amyloid-beta plaques in consecutive sections were then visualized by immunohistochemical [anti-A $\beta$ antibody (clone 6F/3D, diluted 1:100, Dako)] staining for neuropathological confirmation of findings.

\subsubsection{Tumor and control human brain samples}

Brain tumor samples were retrieved intraoperatively. The surgeon used a surgical fluorescence microscope to identify 5-ALA-positive areas and resected the tumor-associated tissue. During tumor resection, 5-ALA-positive and/or -negative tissue samples were routinely collected. Half of the biopsy samples was directly processed for routine neuropathological work-up and the other half was imaged with the multimodal setup (Ethical approval EK 419/2008 - Amendment 04/2018). The fluorescence images were acquired before the OCM volumes to prevent photo bleaching. After OCM and FI, the samples were prepared for histology. Hematoxylin and eosin staining was performed and micrographs were acquired with a slide scanner (Hamamatsu NanoZoomer 2.0 HT). In total, 12 biopsies of 6 patients (age range 47 to 65 years) diagnosed with brain tumors were investigated. According to the surgeon six samples showed strong 5-ALA-positive fluorescence, three samples showed vague 5-ALA-positive fluorescence and three samples were 5-ALA negative. A detailed description of all biopsies can be found in Table 1 .

\subsection{Data Acquisition and Postprocessing}

Processed OCM volumes consisted of $4096(z) \times 500(x) \times$ $500(y)$ pixels and the fluorescence images comprised $500(x) \times$ $500(y)$ pixels. The field of view was $200 \times 200 \mu \mathrm{m}^{2}$. Data were acquired using a custom made Labview program (LabView 2015, Version 15.0, 64-bit, National Instruments). The OCM data were processed following the steps described by Lichtenegger et al., ${ }^{7}$ and en-face projections were generated by averaging intensity over various depths. Fiji was used to generate composition images of FI and OCM results. ${ }^{68}$

\subsubsection{Data processing of the brain biopsy measurements}

An overview over the data processing pipeline for the tumor samples is shown in Fig. 2. After FI [Fig. 2(1)] and OCM [Fig. 2(3)] acquisition, surface flattening was performed, and attenuation maps [Fig. 2(4)] were generated following previous work. ${ }^{6,12,69}$ A region of interest consisting of 100 B-scans was chosen manually and for each B-scan, the average attenuation coefficient was calculated [Fig. 2(5)]. The respective average fluorescence signal [Fig. 2(2)] was calculated to create a scatter plot [Fig. 2(6)]. The fluorescence data $\left[\operatorname{data}_{\mathrm{FI}}(n), n=1 \ldots N\right.$, 
Table 1 The detailed information of the 12 biopsies of 6 patients. The 5-ALA status and the definite diagnosis of patient records are shown. 5-ALA - corresponds to no fluorescence, 5-ALA + to vague, and ++ to strong 5-ALA fluorescence.

\begin{tabular}{|c|c|c|c|}
\hline $\begin{array}{l}\text { Patient } \\
\text { ID }\end{array}$ & $\begin{array}{l}\text { Biopsy } \\
(n)\end{array}$ & $\begin{array}{l}\text { Fluorescence } \\
\text { status }\end{array}$ & Definite diagnosis \\
\hline 1 & 1 & 5-ALA - & Adjacent brain parenchyma \\
\hline 1 & 2 & $5-A L A++$ & $\begin{array}{l}\text { Compact tumor and infiltration } \\
\text { zone }\end{array}$ \\
\hline II & 3 & 5-ALA ++ & $\begin{array}{l}\text { Compact tumor and infiltration } \\
\text { zone }\end{array}$ \\
\hline II & 4 & $5-A L A++$ & $\begin{array}{l}\text { Diffuse tumor infiltration and } \\
\text { necrotic tissue }\end{array}$ \\
\hline III & 5 & $5-A L A++$ & Infiltration zone \\
\hline IV & 6 & 5-ALA - & Adjacent brain parenchyma \\
\hline V & 7 & $5-A L A+$ & $\begin{array}{l}\text { Physiological tissue and } \\
\text { infiltration zone }\end{array}$ \\
\hline v & 8 & $5-A L A+$ & $\begin{array}{l}\text { Physiological tissue and } \\
\text { infiltration zone }\end{array}$ \\
\hline v & 9 & $5-\mathrm{ALA}++$ & Compact metastatic tissue \\
\hline VI & 10 & 5-ALA - & Adjacent brain parenchyma \\
\hline VI & 11 & 5-ALA - & Adjacent brain parenchyma \\
\hline VI & 12 & $5-A L A++$ & $\begin{array}{l}\text { Compact tumor and infiltration } \\
\text { zone }\end{array}$ \\
\hline
\end{tabular}

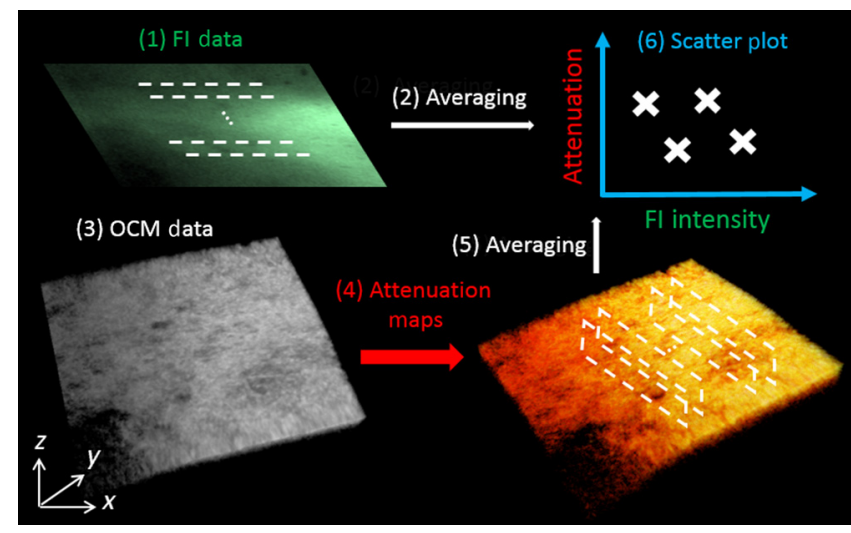

Fig. 2 The data processing pipeline. First, OCM and FI images are acquired. Attenuation maps are generated from the OCM data. Attenuation coefficients are averaged in a chosen region of interest. The results from the attenuation analysis are then compared in a scatter plot with their respective averaged FI values.

$\left(N=12\right.$ biopsies)] was normalized using $\operatorname{norm}_{\mathrm{FI}}(n)=$ $\frac{\operatorname{data}_{\mathrm{FI}}(n)-\min \left(\operatorname{data}_{\mathrm{FI}}\right)}{\max \left(\operatorname{data}_{\mathrm{FI}}\right)-\min \left(\text { data }_{\mathrm{FI}}\right)}$. To calculate the maximum $\left[\max \left(\operatorname{data}_{\mathrm{FI}}\right)\right]$ and minimum $\left[\min \left(\operatorname{data}_{\mathrm{FI}}\right)\right]$, all data sets were used. The resulting clusters (each point represents one B-scan) were visualized in a scatter plot (5-ALA-positive/negative and infiltration zone). For the statistical analysis, mean values of attenuation and FI data were calculated for each of the 12 samples. Mann-Whitney $\mathrm{U}$ tests with Bonferroni correction were performed to test for the equality of the distributions in attenuation and fluorescence data using a significance level of $p<0.01$.

Further cell counting in OCM volumes was performed. Subvolumes consisting of regions of interest of $120 \times 200 \times$ $20 \mu \mathrm{m}^{3}$ were evaluated. For each volume three times the same area was evaluated. The cells, appearing as hyposcattering regions in the intensity volumes, were manually annotated using ITK-Snap. ${ }^{70}$ The binary output files were then used to perform automatic cell counting using the three-dimensional (3-D) objects counter tool in Fiji.

\section{Results}

\subsection{Curcumin Phantom Measurements}

A curcumin phantom was imaged to validate the performance of the system. The excitation and emission spectra of curcumin are shown in Fig. 3(a) along with the filters used. In the fluorescence image [Fig. 3(b)], a clear contrast between pure paper and the mounting medium mixed with curcumin is visible. A representative intensity B-scan image and the intensity averaged OCM en-face projection over $50 \mu \mathrm{m}$ are shown in Figs. 3(c) and 3(d), respectively. Note that it is challenging to distinguish the curcumin from the paper in the OCM en-face image. Figure 3(e) shows an overlay of the OCM en-face image (red) and the corresponding fluorescence image (green).

\subsection{Amyloid-Beta Plaque Imaging in Brain Tissue of an Alzheimer's Disease Mouse Model}

Curcumin-stained brain sections of a mouse model of AD were imaged with the combined setup (Fig. 4). An immunohistochemical and hematoxylin-stained histology image of an adjacent brain region is shown in Fig. 4(a). Amyloid-beta plaques appear as brown structures. Figure 4(b) shows an image of a $3-\mu \mathrm{m}$-thick brain section stained with curcumin taken with a commercial fluorescence Olympus microscope. The plaques can be identified as regions with increased fluorescence due to curcumin. A similar region in a 3- $\mu$ m-thick brain section was imaged with the OCM/FI setup, and the FI results are shown in Fig. 4(c). Again, amyloid-beta plaques can be identified as highly fluorescent structures. A $100-\mu \mathrm{m}$-thick brain slice was first imaged with a commercial laser scanning microscope [Fig. 4(d)]. A representative OCM B-scan of the vibratome section is shown in Fig. 4(e). A plaque can be identified as a hyperscattering structure. Figure 4(f) shows an OCM intensity en-face projection over $10 \mu \mathrm{m}$ underneath the tissue surface. A plaque shows up as a highly scattering feature. The corresponding fluorescence image is shown in Fig. 4(g). The focus was set at the same depth as for the OCM acquisition. Finally, a composite image of OCM (red) and fluorescence (green) image is shown in Fig. 4(h). The amyloid-beta plaque, which is present in both the OCM and the fluorescence image, can be observed in a yellowish color. Another plaque, which is present in the fluorescence image, is barely visible in the OCM image. The dark spots in the fluorescence image are cells and appear as hyposcattering in the OCM images. Figure 4(i) shows an average en-face projection over the whole $100 \mu \mathrm{m}$ in a slightly bigger area with hyperscattering plaques indicated by yellow arrows.

\subsection{Imaging of 5-ALA Brain Tumor Biopsies}

5-ALA-positive tumor samples retrieved intraoperatively were imaged prior to routine neuropathologic work-up. In Fig. 5(a), 


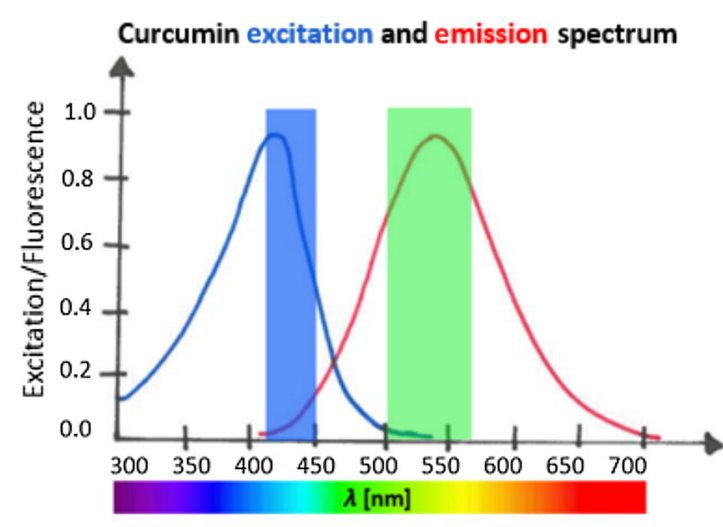

(a)

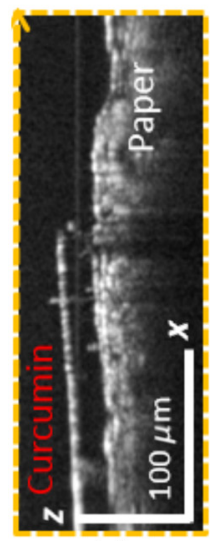

(c)

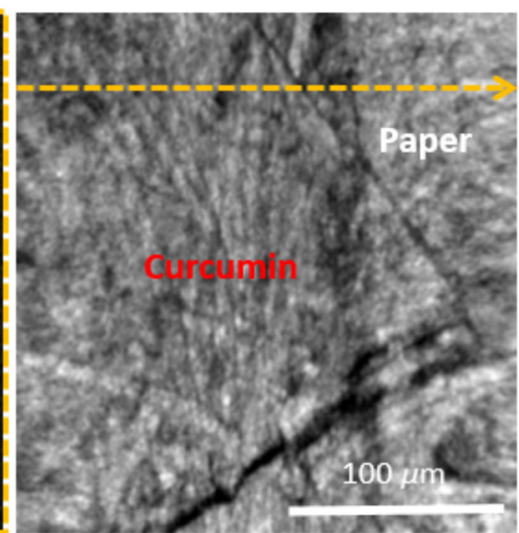

(d)

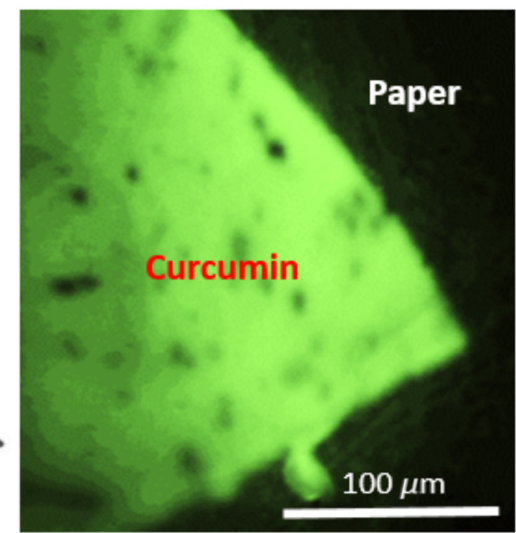

(b)

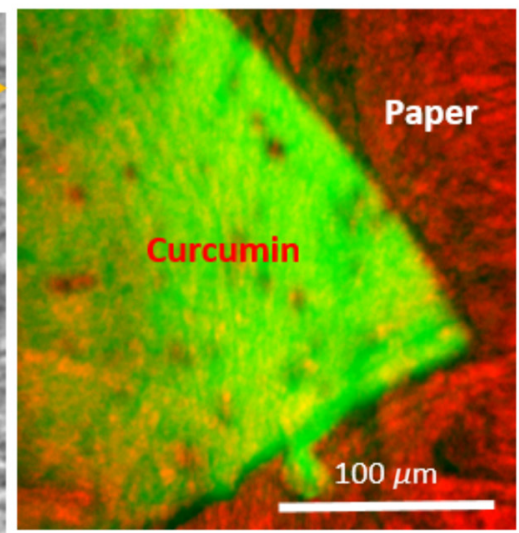

(e)

Fig. 3 Curcumin phantom imaging with the multimodal setup. (a) Excitation and emission spectra of curcumin. The excitation (blue) and emission (green) filters used in the system are indicated by color bands. ${ }^{71}$ (b) Fluorescence image of the mounting medium mixed with curcumin on the paper. (c) A corresponding B-scan OCM image taken at the location indicated by a yellow dashed line in image (d). (d) OCM en-face projection over $50 \mu \mathrm{m}$. (e) Overlay of the OCM (red) and the fluorescence image (green). All images are taken with a $20 \times$ magnification objective lens.

the excitation and emission spectra of 5-ALA are plotted. The emission (red) and excitation (blue) filter spectra are indicated by color bands. ${ }^{72}$ In Figs. 5(b)-5(d), histological images are shown for a 5-ALA-negative area, an infiltration zone, and a 5-ALA-positive area. Tumor area and infiltration zone can be distinguished by the densities of malignant cells. In the infiltration zone, areas of physiological brain tissue with infiltrating malignant cells (indicated by green arrows) can be observed. An average en-face projection over $20 \mu \mathrm{m}$ and a representative B-scan image of a 5-ALA-positive area are shown in Figs. 5(e) and 5(f), respectively. The averaged attenuation map shows lower values in the left lower corner [Fig. 5(g)]. The combined OCM (blue) and fluorescence image (red) in Fig. 5(h), in the same area, shows a stronger fluorescence signal indicating a higher density of malignant cells. ${ }^{73}$ An average en-face projection over $20 \mu \mathrm{m}$ and a representative B-scan image of a 5-ALAnegative area are shown in Figs. 5(i) and 5(j), respectively. Compared to 5-ALA-positive tissue, the tissue morphology is more homogeneous and the intensity in the OCM image is increased.

Cell counting was conducted in three OCM data sets, three times each for one tumor area, a nontumorous area, and an infiltration zone. As shown in Fig. 6(a), the cell count in the 5-ALApositive area and the infiltration zone was $139 \%$ and $15 \%$ higher than in 5-ALA-negative tissue, respectively. Attenuation maps for all measured OCM volumes were calculated. Figure 6(b) shows the averaged attenuation coefficients over B-scans plotted over the averaged intensity values retrieved from the fluorescence images. For the statistical evaluation mean values for all 12 data sets were evaluated. The 5-ALA-positive tissue exhibits lower attenuation coefficients and higher fluorescence values when compared to 5-ALA-negative area. The mean attenuation values in the infiltration zone compared to the 5-ALA-negative area showed a trend to be lower but no significance was found $(p=0.014)$. The mean attenuation values in the infiltration zone compared to the 5-ALA-positive area showed a trend to be higher but no significance was found $(p=0.15)$. All other results were statistically significant (Table 2) with a $p$-value of $<0.01$. The mean values of the clusters which were tested for different distributions are indicated by the color bars in Fig. 6(b).

\section{Discussion}

We developed a combined visible light OCM and FI setup. Our multimodal system presents a compact and simple design to consecutively acquire OCM and FI data by using a single light source for both modalities in comparison to previously reported work. ${ }^{15,18,19,22-24,26-33}$ Switching from OCM imaging to FI is achieved by simply flipping two mirrors in the setup. Another advantage of our setup is that it has the possibility to detect a 


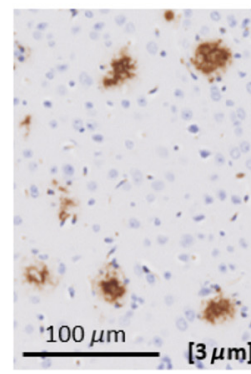

(a)

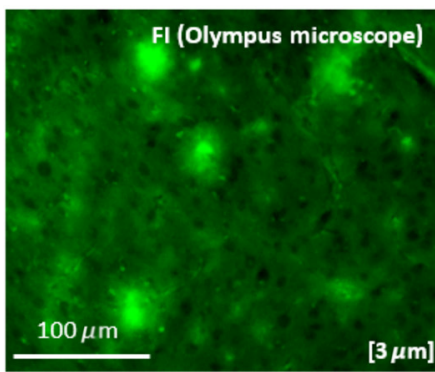

(b)

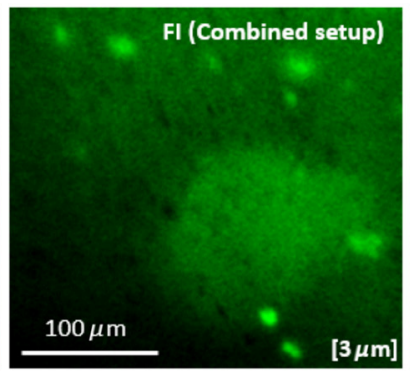

(c)

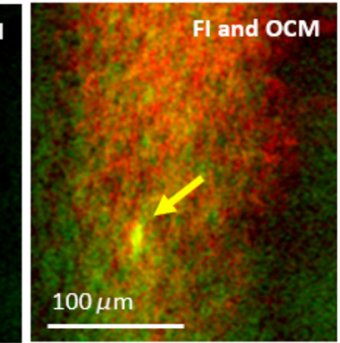

(h)

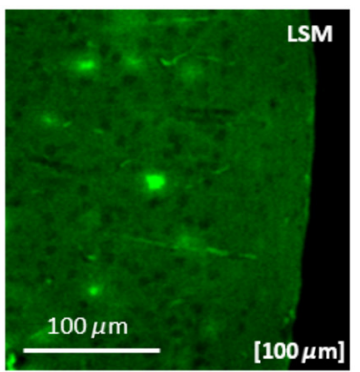

(d)

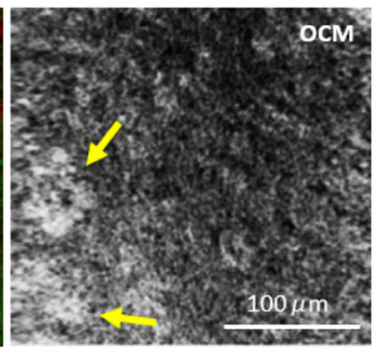

(i)

(e)

(f)

(g)

Fig. 4 Imaging of curcumin-stained brain sections of an AD mouse model. (a) Immunohistochemical and hematoxylin-stained tissue section of a plaque-rich region (20× magnification). (b) Fluorescence image of the 3- $\mu$ m-thick section imaged with 20x magnification (Olympus BX51 microscope). (c) FI image taken with the combined OCM/FI setup of a 3- $\mu \mathrm{m}$-thick section. (d) The $100-\mu \mathrm{m}$-thick brain section imaged with the laser scanning microscope (LSM 700 Zeiss). (e) Representative OCM intensity B-scan image. (f) OCM en-face projection over $10 \mu \mathrm{m}$ underneath the tissue surface. (g) Corresponding fluorescence image. (h) An overlay of the OCM (red) and the fluorescence image (green). (i) OCM en-face projection over the whole depth range. The thicknesses of the measured tissue slices are indicated in brackets in the right corner of the images. Plaques are marked with yellow arrows in images (f)-(i).

variety of standard fluorescence dyes as the light source spectrum covers the whole visible range. To image specific fluorescent dyes, the system can be equipped with different filter cubes that are readily available for commercial fluorescence microscopes. In order to further improve the system and to enable fast switching between different dyes, it might be an option to assemble multiple filter cubes that could then be integrated in an automated wheel or stage for quick exchange. At the same time, using the broad visible spectrum for OCM, an axial resolution of $0.88 \mu \mathrm{m}$ in brain tissue was achieved, which enabled imaging on a cellular level. When investigating amyloid-beta plaques, which are in the range of 10 to $200 \mu \mathrm{m}$, a high resolution is crucial. ${ }^{74}$

Further technological improvements of the OCM/FI setup could enable dynamic focusing and 3-D FI. While focusing was done manually in the current implementation, setting the focus for FI and OCM imaging in the future could be done using an automatic $z$-stage. The implementation of such a stage would also enable the acquisition of confocal image stacks of 3-D FI data. For now, the focus was set at the same position as the OCM image, at the top of the surface of the tissue. The penetration depth into brain tissue using visible light OCM was limited to $\sim 100 \mu \mathrm{m}$. Investigating optically cleared tissue would increase the penetration depth, as has been shown in our previous work. ${ }^{6}$ Further, to achieve good FI results, a flat surface would be optimal. Imaging a curved tissue surface was challenging as the signal at the image borders is reduced and fewer structures can be identified. This effect can for example be observed in Figs. 4(f)-4(h). One possibility to overcome this issue could be to acquire multiple stacks and fuse them to increase the image quality and suppress speckle noise. ${ }^{75}$
A commercial $20 \times$ objective lens was used for imaging. Using higher numerical aperture (NA) would result in a better fluorescence signal. In future, objective lenses with even higher NA will be used to investigate smaller anatomical features with a better fluorescent response.

For the data acquisition, first the FI image and then OCM volumes were acquired to prevent bleaching. For all measurements, the same power, pinhole settings, and objective lens were used. The focus was always set at the tissue surface. Furthermore, imaging was performed in a darkened room. The measured FI intensity is also dependent on the biopsy shape and the autofluorescence. ${ }^{76}$ As a next step calculating a relative or quantitative fluorescence intensity will be investigated, following, for example, Valdés et al. ${ }^{77}$ For that purpose, a fluorescence phantom is needed as a reference to achieve correct relative intensity values.

Two application possibilities of the setup in the field of neuroimaging were presented. First, brain tissue slices of an AD mouse model stained with curcumin were imaged. The investigations revealed that with the same light source amyloid-beta plaques can be specifically identified using FI, and the morphology of the brain tissue, including anatomical features, can be investigated by OCM. The plaques were identified in the OCM images as highly scattering structures and in the FI images as highly fluorescent spots. The additional fluorescence channel confirmed that the highly scattering structures observed with OCM are amyloid-beta plaques. The results were in good agreement with literature and histology. ${ }^{5,25}$ To improve the sensitivity to detect amyloid-beta plaques, thioflavin-S-stained brain tissue could be investigated. It was shown that thioflavin-S may be a more suitable fluorescence marker for the detection of 


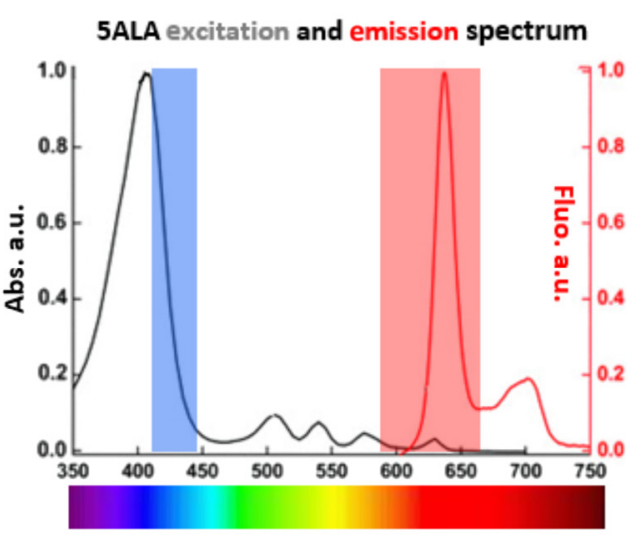

(a)

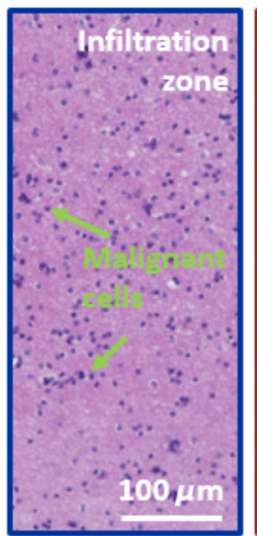

(c)

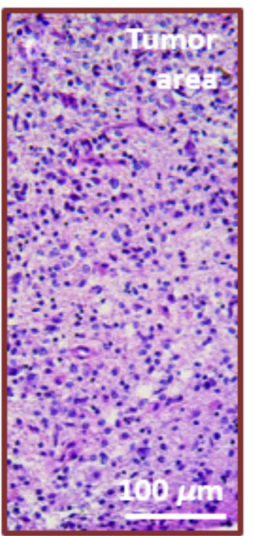

(d)

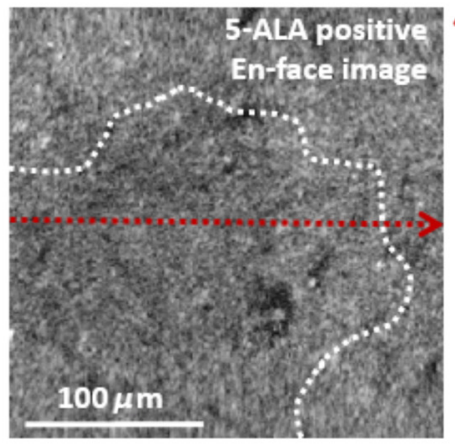

(e)

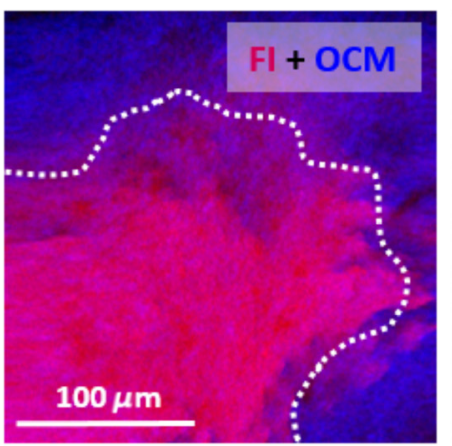

(h)

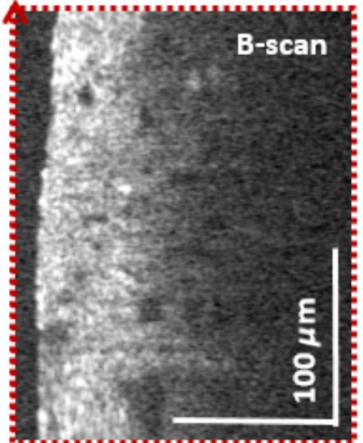

(f)

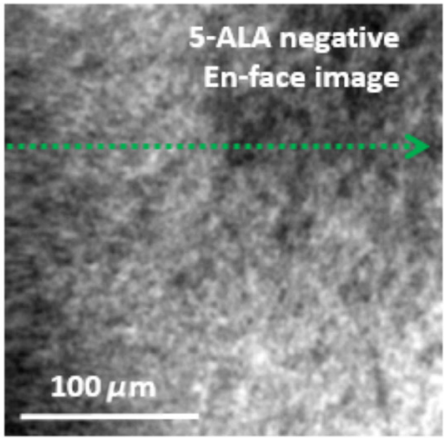

(i)

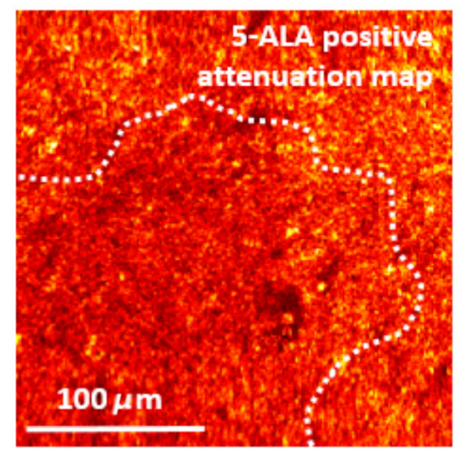

(g)

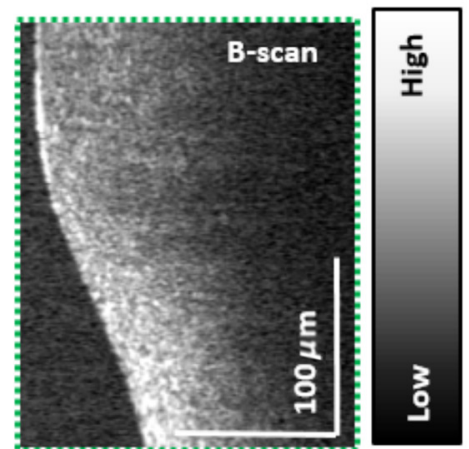

(j)

Fig. 5 Imaging of 5-ALA-positive and -negative human brain biopsies. (a) Excitation and emission spectra of 5-ALA . ${ }^{72}(b-d)$ Histology of a control region, an infiltration zone, and a core tumor zone. (e) Intensity en-face OCM projection over $20 \mu \mathrm{m}$ in a 5-ALA-positive area. (f) Representative B-scan image of (e). (g) Average attenuation map over $20 \mu \mathrm{m}$ in a 5-ALA-positive area. (h) OCM en-face projection (blue) overlaid with the Fl image (red). The dotted line in panels (e) and $(\mathrm{g})$ outlines the hyperfluorescent region in panel (h). (i) Intensity en-face OCM projection over $20 \mu \mathrm{m}$ in a 5-ALA-negative area. (j) Representative B-scan image of (i).

amyloid-beta plaques compared to curcumin. ${ }^{46}$ As a next step, the FI channel could be utilized to investigate tau accumulations, which could add additional insights in the mechanisms of the disease.

Second, 5-ALA-positive and -negative human brain biopsies from tumor surgeries were investigated prior to neuropathologic work-up. The 5-ALA-positive regions in the samples were visualized by FI and morphological OCM information of the tissue was acquired with minimal delay. For all data sets, attenuation maps were calculated and analyzed. The attenuation in the 5-ALA-positive areas was significantly lower than 5-ALAnegative brain areas, and the fluorescence signal was significantly higher. Due to autofluorescence of the tissue, in some cases [see Fig. 6(b)], it was hard to distinguish the negative and infiltration zone. In these cases, the multimodal approach using fluorescence and attenuation values may provide better contrast. Using in total 12 biopsies, a preliminary analysis is presented in this article; however, a greater sample size will be needed to achieve a more reliable statistical conclusion. Still, the attenuation values and their decrease in tumorous tissue were in good agreement with the literature. ${ }^{12,78,79}$ Kut et al. described that tumors infiltrate into white matter, breaking down myelin and therefore decreasing its expression. This ultimately leads to a lower attenuation. ${ }^{12}$ OCM-based cell counting results showed an increase of $139 \%$ from 5-ALA-negative to 5-ALA-positive tissues and $15 \%$ increase in the infiltration zone. Our results 
(a)

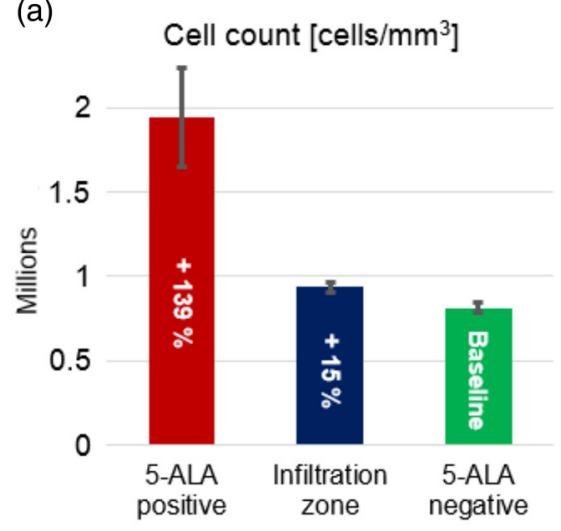

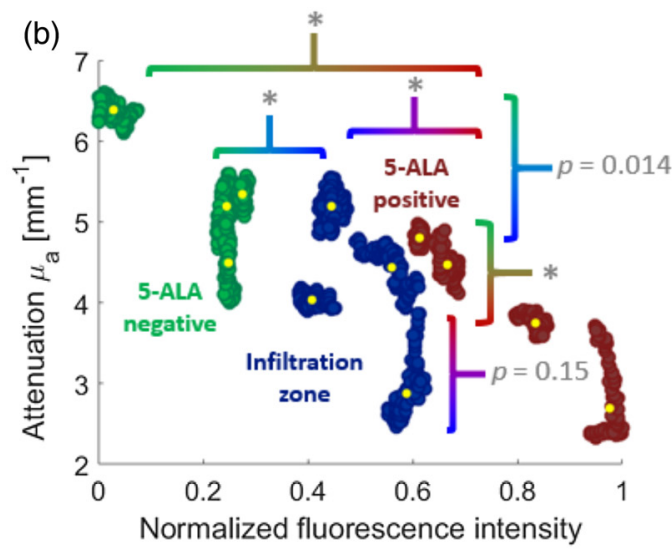

Fig. 6 Evaluation of OCM and FI data 5-ALA-positive and -negative human brain biopsies. (a) Cell counting results from the OCM volumes. (b) The averaged attenuation coefficients (each point represents one $\mathrm{B}$-scan) were plotted over the averaged intensity values retrieved from the FI images. (*Significance level $p<0.01$.) The mean value of each data set is indicated by a yellow dot.

Table 2 The detailed information of the Mann-Whitney $U$ results, testing if the clusters originated from different continuous distributions. The first column indicates the tests which were conducted, F(5-ALA-) corresponds to the 5-ALA-negative area, $F(5-A L A+)$ to the infiltration zone, and F(5-ALA ++) to the 5-ALA-positive area, and $\mu_{a}$ is indicating the calculated mean attenuation values. ( ${ }^{\star}$ Significance level $p<0.01$.)

\begin{tabular}{lc} 
Mann-Whitney U test & Significance \\
\hline F(5-ALA ++) versus F(5-ALA +) & $p<0.01$ \\
F(5-ALA ++) versus F(5-ALA -) & $p<0.01$ \\
F(5-ALA +) versus F(5-ALA -) & $p<0.01$ \\
$\mu_{a}(5-\mathrm{ALA}++)$ versus $\mu_{a}(5-\mathrm{ALA}+)$ & $p=0.15$ \\
$\mu_{a}(5-\mathrm{ALA}++)$ versus $\mu_{a}(5-\mathrm{ALA}-)$ & $p<0.01$ \\
$\mu_{a}(5-\mathrm{ALA}+)$ versus $\mu_{a}(5-\mathrm{ALA}-)$ & $p<0.014$ \\
\hline
\end{tabular}

showed that malignant tissue exhibits a higher cellularity compared to nontumorous brain tissue. ${ }^{80}$ These data were in agreement with histology. The cell counting results will further be compared to cellularity maps generated from histological data of the same region. OCM findings identifying malignant areas within brain biopsies were further correlated to histopathological routine analysis and were in good agreement. For the future, more samples will be imaged with our multimodal system to evaluate sensitivity and specificity of using OCM/FI for tumor intraoperative assessment. The focus will also lie in differentiating low and high grade glioma samples using the multimodal approach.

\section{Conclusion}

A multimodal visible light OCM and FI setup was introduced. A supercontinuum laser emitting a broad visible spectrum enabled to achieve submicrometer axial resolution for OCM imaging and by simply flipping two mirrors, the system can be changed from OCM to FI mode using the same light source. The performance was characterized by imaging fluorescent microbeads and a curcumin fluorescence phantom. Two applications of the multimodal approach in the field of neuroimaging were demonstrated.
First, curcumin-stained brain slices of a mouse model of AD were imaged. Amyloid-beta plaques, one hallmark of AD, were specifically identified using FI. Consecutively, the brain morphology was investigated in 3-D using OCM, and by FI, the OCM results could be validated. Second, 5-ALA-positive and -negative brain biopsies were imaged. Average attenuation coefficients and fluorescence intensity values showed significances differences between 5-ALA-positive and -negative brain tissue. Cell counting was performed in the OCM data showing a typical increase in cellularity in malignant tissue. This multimodal approach offers the possibility to investigate microscopic, 3-D features using OCM and at the same time gain tissue-specific contrast by FI. Using OCM in combination with FI may therefore be a versatile and powerful tool for many applications in the field of neuroscience.

\section{Disclosures}

None.

\section{Acknowledgments}

The authors thank Andreas Hodul and Franz Beck for their technical support, Tamara Prusa and Theresia Lengheimer for the animal handling, and Günther Regelsberger and Zoe Neuburger for their support during sample preparation and tissue clearing. A special thanks to Thomas Rötzer for his expertise in neuropathology and brain tumors. We also want to thank Gerda Ricken and the neuropathology lab team and Gabor G. Kovacs for sharing their expertise in Alzheimer's disease and brain pathology. We particularly acknowledge Marco Andreana, Andreas Wartak, Florian Beer, Laurin Ginner, Michael Niederleithner, Mikael Erkkilae, and Stanislava Fialová, for their support while setting up the OCM system and performing the measurements. This work was funded by the European Research Council (ERC StG 640396 OPTIMALZ).

\section{References}

1. W. Drexler and J. G. Fujimoto, Optical Coherence Tomography: Technology and Applications, Springer Science \& Business Media, Cham, New York (2015).

2. G. Liu and Z. Chen, "Optical coherence tomography for brain imaging," Prog. Opt. Sci. Photonics 5, 157-172 (2013). 
3. T. Gambichler et al., "Applications of optical coherence tomography in dermatology," J. Dermatol. Sci. 40(2), 85-94 (2005).

4. M. J. Gora et al., "Endoscopic optical coherence tomography: technologies and clinical applications," Biomed. Opt. Express 8(5), 2405-2444 (2017).

5. P. J. Marchand et al., "Visible spectrum extended-focus optical coherence microscopy for label-free sub-cellular tomography," Biomed. Opt. Express 8(7), 3343-3359 (2017).

6. A. Lichtenegger et al., "Spectroscopic imaging with spectral domain visible light optical coherence microscopy in Alzheimer's disease brain samples," Biomed. Opt. Express 8(9), 4007-4025 (2017).

7. A. Lichtenegger et al., "Assessment of pathological features in Alzheimer's disease brain tissue with a large field-of-view visible-light optical coherence microscope," Neurophotonics 5(3), 035002 (2018).

8. P. Albrecht et al., "Optical coherence tomography in parkinsonian syndromes," PLoS ONE 7(4), e34891 (2012).

9. P. Sengupta et al., "Optical coherence tomography findings in patients of Parkinson's disease: an Indian perspective," Ann. Indian Acad. Neurol. 21(2), 150-155 (2018).

10. H. Böhringer et al., "Time-domain and spectral-domain optical coherence tomography in the analysis of brain tumor tissue," Lasers Surg. Med. 38(6), 588-597 (2006).

11. O. Assayag et al., "Imaging of non-tumorous and tumorous human brain tissues with full-field optical coherence tomography," NeuroImage Clin. 2, 549-557 (2013).

12. C. Kut et al., "Detection of human brain cancer infiltration ex vivo and in vivo using quantitative optical coherence tomography," Sci. Transl. Med. 7(292), 292ra100 (2015).

13. W. Drexler et al., "Optical coherence tomography today: speed, contrast, and multimodality," J. Biomed. Opt. 19(7), 071412 (2014).

14. O. M. Ogundele et al., "Basic principles of fluorescence microscopy," World J. Young Researchers 17(1), 17-20 (2013).

15. E. Beaurepaire et al., "Combined scanning optical coherence and twophoton-excited fluorescence microscopy," Opt. Lett. 24(14), 969-971 (1999).

16. Y. Pan et al., "Enhancing early bladder cancer detection with fluorescence-guided endoscopic optical coherent tomography," Opt. Lett. 28(24), 2485-2487 (2003).

17. Z. Wang et al., "Fluorescence guided optical coherence tomography for the diagnosis of early bladder cancer in a rat model," J. Urol. 174(6), 2376-2381 (2005).

18. S. Tang et al., "Combined multiphoton microscopy and optical coherence tomography using a 12 -fs broadband source," J. Biomed. Opt. 11(2), 020502 (2006).

19. C. Vinegoni et al., "Integrated structural and functional optical imaging combining spectral-domain optical coherence and multiphoton microscopy," Appl. Phys. Lett. 88(5), 053901 (2006).

20. L. P. Hariri et al., "Ex vivo optical coherence tomography and laserinduced fluorescence spectroscopy imaging of murine gastrointestinal tract," Comp. Med. 57(2), 175-185 (2007).

21. P. M. Andrews et al., "High-resolution optical coherence tomography imaging of the living kidney," Lab. Invest. 88(4), 441-449 (2008).

22. J. Park et al., "A dual-modality optical coherence tomography and fluorescence lifetime imaging microscopy system for simultaneous morphological and biochemical tissue characterization," Biomed. Opt. Express 1(1), 186-200 (2010).

23. S. Yuan et al., "Co-registered optical coherence tomography and fluorescence molecular imaging for simultaneous morphological and molecular imaging," Phys. Med. Biol. 55(1), 191-206 (2009).

24. E. Auksorius et al., "Dual-modality fluorescence and full-field optical coherence microscopy for biomedical imaging applications," Biomed. Opt. Express 3(3), 661-666 (2012).

25. T. Bolmont et al., "Label-free imaging of cerebral $\beta$-amyloidosis with extended-focus optical coherence microscopy," J. Neurosci. 32(42), 14548-14556 (2012).

26. N. Iftimia et al., "Fluorescence-guided optical coherence tomography imaging for colon cancer screening: a preliminary mouse study," Biomed. Opt. Express 3(1), 178-191 (2012).

27. D. Lorenser et al., "Dual-modality needle probe for combined fluorescence imaging and three-dimensional optical coherence tomography," Opt. Lett. 38(3), 266-268 (2013).
28. M. Jiang et al., "Simultaneous optical coherence tomography and lipofuscin autofluorescence imaging of the retina with a single broadband light source at $480 \mathrm{~nm}, "$ Biomed. Opt. Express 5(12), 4242-4248 (2014).

29. C. Berclaz et al., "Combined optical coherence and fluorescence microscopy to assess dynamics and specificity of pancreatic beta-cell tracers," Sci. Rep. 5, 10385 (2015).

30. R. P. McNabb et al., "Method for single illumination source combined optical coherence tomography and fluorescence imaging of fluorescently labeled ocular structures in transgenic mice," Exp. Eye Res. 151, 68-74 (2016).

31. Z. Nafar et al., "Visible-light optical coherence tomography-based multimodal retinal imaging for improvement of fluorescent intensity quantification," Biomed. Opt. Express 7(9), 3220-3229 (2016).

32. O. Thouvenin, M. Fink, and A. C. Boccara, "Dynamic multimodal fullfield optical coherence tomography and fluorescence structured illumination microscopy," J. Biomed. Opt. 22(2), 026004 (2017).

33. H. S. Nam et al., "Multispectral analog-mean-delay fluorescence lifetime imaging combined with optical coherence tomography," Biomed. Opt. Express 9(4), 1930-1947 (2018).

34. M. J. Ju et al., "Visible light sensorless adaptive optics for retinal structure and fluorescence imaging," Opt. Lett. 43(20), 5162-5165 (2018).

35. F. Feroldi et al., "High resolution combined molecular and structural optical imaging of colorectal cancer in a xenograft mouse model," Biomed. Opt. Express 9(12), 6186-6204 (2018).

36. J. Yi et al., "Visible-light optical coherence tomography for retinal oximetry," Opt. Lett. 38(11), 1796-1798 (2013).

37. S. P. Chong et al., "Ultrahigh resolution retinal imaging by visible light OCT with longitudinal achromatization," Biomed. Opt. Express 9(4), 1477-1491 (2018).

38. D. J. Harper et al., "White light polarization sensitive optical coherence tomography for sub-micron axial resolution and spectroscopic contrast in the murine retina," Biomed. Opt. Express 9(5), 2115-2129 (2018).

39. B. Povazay et al., "Submicrometer axial resolution optical coherence tomography," Opt. Lett. 27(20), 1800-1802 (2002).

40. X. Shu, L. J. Beckmann, and H. F. Zhang, "Visible-light optical coherence tomography: a review," J. Biomed. Opt. 22(12), 121707 (2017).

41. Alzheimer's Association, "2018 Alzheimer's disease facts and figures," Alzheimer's Dementia 14(3), 367-429 (2018).

42. D. M. Wilcock, M. N. Gordon, and D. Morgan, "Quantification of cerebral amyloid angiopathy and parenchymal amyloid plaques with Congo red histochemical stain," Nat. Protocols 1(3), 1591-1595 (2006).

43. T. van Groen, A. J. Kiliaan, and I. Kadish, "Deposition of mouse amyloid $\beta$ in human APP/PS1 double and single AD model transgenic mice," Neurobiol. Dis. 23(3), 653-662 (2006).

44. M. Garcia-Alloza et al., "Curcumin labels amyloid pathology in vivo, disrupts existing plaques, and partially restores distorted neurites in an Alzheimer mouse model," J. Neurochem. 102(4), 1095-1104 (2007).

45. W. E. Klunk et al., "Uncharged thioflavin-T derivatives bind to amyloidbeta protein with high affinity and readily enter the brain," Life Sci. 69(13), 1471-1484 (2001).

46. J. den Haan et al., "Different curcumin forms selectively bind fibrillar amyloid beta in post mortem Alzheimer's disease brains: Implications for in-vivo diagnostics," Acta Neuropathol. Commun. 6(1), 75 (2018).

47. G. M. Cole et al., "NSAID and antioxidant prevention of Alzheimer's disease: lessons from in vitro and animal models," Ann. New York Acad. Sci. 1035(1), 68-84 (2004).

48. R. W. Rand and P. J. Jannetta, "Microneurosurgery: application of the binocular surgical microscope in brain tumors, intracranial aneurysms, spinal cord disease, and nerve reconstruction," Neurosurgery 15(1), 319-342 (1968).

49. R. Stupp et al., "Radiotherapy plus concomitant and adjuvant temozolomide for glioblastoma," N. Engl. J. Med. 352(10), 987-996 (2005).

50. F. E. Bleeker, R. J. Molenaar, and S. Leenstra, "Recent advances in the molecular understanding of glioblastoma," J. Neuro-Oncol. 108(1), 11-27 (2012).

51. M. J. Colditz, K. van Leyen, and R. L. Jeffree, "Aminolevulinic acid (ALA)-protoporphyrin IX fluorescence guided tumour resection. Part 2: Theoretical, biochemical and practical aspects," J. Clin. Neurosci. 19(12), 1611-1616 (2012). 
52. G. Widhalm, "Intra-operative visualization of brain tumors with 5-aminolevulinic acid-induced fluorescence," Clin. Neuropathol. 33(4), 260-278 (2014).

53. G. Widhalm et al., "5-aminolevulinic acid is a promising marker for detection of anaplastic foci in diffusely infiltrating gliomas with nonsignificant contrast enhancement," Cancer 116(6), 1545-1552 (2010).

54. S. Kaneko and S. Kaneko, "Fluorescence-guided resection of malignant glioma with 5-ALA," Int. J. Biomed. Imaging 2016, 1-11 (2016).

55. H. Böhringer et al., "Imaging of human brain tumor tissue by nearinfrared laser coherence tomography," Acta Neurochir. 151(5), 507-517 (2009).

56. B. J. Vakoc et al., "Cancer imaging by optical coherence tomography: preclinical progress and clinical potential," Nat. Rev. Cancer 12(5), 363-368 (2012).

57. K. S. Yashin et al., "Multimodal optical coherence tomography for in vivo imaging of brain tissue structure and microvascular network at glioblastoma," Proc. SPIE 10050, $100500 \mathrm{Z}$ (2017).

58. K. Yashin et al., "Visual assessment criteria of microstructural ex vivo co- and cross-polarized optical coherence tomography images in gliomas," Proc. SPIE 10685, 106853F (2018).

59. T. Garzon-Muvdi et al., "Intraoperative imaging techniques for glioma surgery," Future Oncol. 13(19), 1731-1745 (2017).

60. M. Finke et al., "Automatic scanning of large tissue areas in neurosurgery using optical coherence tomography," Int. J. Med. Rob. Comput. Assisted Surg. 8(3), 327-336 (2012).

61. J. P. Ehlers et al., "Integrative advances for OCT-guided ophthalmic surgery and intraoperative OCT: microscope integration, surgical instrumentation, and heads-up display surgeon feedback," PLOS ONE 9(8), e105224 (2014).

62. M. Lara-Velazquez et al., "Advances in brain tumor surgery for glioblastoma in adults," Brain Sci. 7(12), 166 (2017).

63. J. Binding et al., "Brain refractive index measured in vivo with high-NA defocus-corrected full-field OCT and consequences for two-photon microscopy," Opt. Express 19(6), $4833-4847$ (2011).

64. R. Reiserer et al., "Impaired spatial learning in the APPSwe+ PSEN1 eltaE9 bigenic mouse model of Alzheimer's disease," Genes Brain Behav. 6(1), 54-65 (2007).

65. J. L. Jankowsky et al., "Mutant presenilins specifically elevate the levels of the 42 residue $\beta$-amyloid peptide in vivo: evidence for augmentation of a 42-specific $\gamma$ secretase," Hum. Mol. Genet. 13(2), 159-170 (2003).

66. J. L. Jankowsky et al., "Co-expression of multiple transgenes in mouse CNS: a comparison of strategies," Biomol. Eng. 17(6), 157-165 (2001).

67. E. Murray et al., "Simple, scalable proteomic imaging for highdimensional profiling of intact systems," Cell 163(6), 1500-1514 (2015).

68. J. Schindelin et al., "Fiji: an open-source platform for biological-image analysis," Nat. Methods 9(7), 676-682 (2012).

69. K. Vermeer et al., "Depth-resolved model-based reconstruction of attenuation coefficients in optical coherence tomography," Biomed. Opt. Express 5(1), 322-337 (2014).

70. P. A. Yushkevich et al., "User-guided 3D active contour segmentation of anatomical structures: significantly improved efficiency and reliability," Neuroimage 31(3), 1116-1128 (2006).

71. M. Condat et al., "Photoinduced curcumin derivative-coatings with antibacterial properties," RSC Adv. 5(104), 85214-85224 (2015).

72. R. M. Valentine et al., "Modelling fluorescence in clinical photodynamic therapy," Photochem. Photobiol. Sci. 12(1), 203-213 (2013).

73. W. Stummer et al., "5-aminolevulinic acid-derived tumor fluorescence: the diagnostic accuracy of visible fluorescence qualities as corroborated by spectrometry and histology and postoperative imaging," Neurosurgery 74(3), 310-320 (2013).

74. M. LaFontaine and M. Cumbay, "Focus: the aging brain: amyloid-beta Alzheimer targets: protein processing, lipid rafts, and amyloid-beta pores," Yale J. Biol. Med. 89(1), 5 (2016).

75. V. J. Srinivasan et al., "Optical coherence microscopy for deep tissue imaging of the cerebral cortex with intrinsic contrast," Opt. Express 20(3), 2220-2239 (2012).

76. J. C. Waters, "Accuracy and precision in quantitative fluorescence microscopy," J. Cell Biol. 185(7), 1135-1148 (2009).

77. P. A. Valdés et al., "Quantitative, spectrally-resolved intraoperative fluorescence imaging," Sci. Rep. 2, 798 (2012).
78. W. Yuan et al., "Robust and fast characterization of OCT-based optical attenuation using a novel frequency-domain algorithm for brain cancer detection," Sci. Rep. 7, 44909 (2017).

79. K. S. Yashin et al., "Quantitative nontumorous and tumorous human brain tissue assessment using microstructural co-and cross-polarized optical coherence tomography," Sci. Rep. 9(1), 2024 (2019).

80. T. Roetzer et al., "Evaluating cellularity and structural connectivity on whole brain slides using a custom-made digital pathology pipeline," J. Neurosci. Methods 311, 215-221 (2019).

Antonia Lichtenegger received her master's degrees in technical mathematics and biomedical engineering from the Technical University of Vienna in 2014 and 2015, respectively. She is currently a PhD candidate in the Center for Medical Physics and Biomedical Engineering at the Medical University of Vienna. Her interests include designing and setting up optical coherence microscopy systems and image processing in biomedical optics and neuroscience.

Johanna Gesperger received her master's degree in medical technology from the University of Applied Sciences Wiener Neustadt in 2017. She is currently enrolled as a PhD student at the Medical University of Vienna. Her main research interest is the assessment of the heterogeneity of primary brain tumors with different methods, focusing on optical imaging, digital pathology, and molecular methods.

Barbara Kiesel is a resident for neurosurgery at the Medical University Vienna and a PhD student of the Doctoral Programme for Clinical Neuroscience. Her research focus is on neuro-oncology and techniques for intraoperative visualization of brain tumors.

Martina Muck received her BSc in biomedical science from the University of Applied Sciences FH Campus Wien and finished her master's program for tissue engineering and regenerative medicine at the University of Applied Sciences Technikum in Vienna. She is working on the preparation of brain samples for OCM imaging and histology.

Pablo Eugui finished his master's degree in biomedical engineering at the Universidad Publica de Navarra in Spain in 2015. He is currently enrolled as a PhD student of the Medical University of Vienna and is working on fiber-based optical coherence tomography systems. His interests are optical imaging with different techniques, signal and image processing applied to the medical field, and the study of different neurological diseases.

Danielle J. Harper received her master's degree in physics from the University of St. Andrews in the United Kingdom in 2015. As a PhD student at the Medical University of Vienna, she is currently working to design a new OCT system that is tailored for the in-vivo functional imaging of the retina of animal models. Her main interests lie in optical imaging, particularly in the optical designs which make techniques such as OCT possible.

Matthias Salas is a post-doc at the Center for Medical Physics and Biomedical Engineering at the Medical University of Vienna. He studied civil engineering specialized in electricity at the Universidad Católica de Chile. He received his master's degree in optics at the École Polytechnique in Paris and received his PhD in medical physics from the Medical University of Vienna in 2018. His research interests are the development of new optical devices for ophthalmic applications, particularly adaptive optics OCT and applications for improved visualization of diseases in clinical research.

Marco Augustin received his master's degree in medical informatics from Vienna University of Technology in 2014 and his PhD in medical physics from Medical University of Vienna in 2018, respectively. Currently, he is enrolled as a postdoctoral associate at the Center for Medical Physics and Biomedical Engineering at the Medical University of Vienna. His interests include optical imaging techniques, especially optical coherence tomography and its functional extensions, as well as image processing and pattern recognition, particularly in life sciences.

Conrad W. Merkle received his $\mathrm{PhD}$ in biomedical engineering from the University of California, Davis, USA, in 2018, he joined the Baumann Lab in February 2018 as a postdoctoral researcher. His 
main research interests are focused on the development and application of optical imaging techniques, specifically optical coherence tomography (OCT), to study biomedical systems for preclinical or clinical research. In particular, he is interested in the role of microvascular hemodynamics in the progression of various brain and eye diseases.

Christoph K. Hitzenberger is a professor of medical physics, vicechair of the Center for Medical Physics and Biomedical Engineering at the Medical University of Vienna, and editor-in-chief of Biomedical Optics Express. He has worked in biomedical optics since 1987 and is one of the pioneers of low coherence ocular biometry and OCT. He is a fellow of SPIE and OSA. His pioneering contributions to OCT were awarded with the Russ Prize of the National Academy of Engineering in 2017.

Georg Widhalm is an associate professor for neurosurgery from the Medical University Vienna and is an expert for surgery of brain tumors. Currently, he is the chair of the Tumor Section of the Austrian
Neurosurgical Society. His main research area is the development of innovative techniques to visualize brain and spinal tumors with assistance of intraoperative fluorescence. Recently, he completed a research stay at the University of California, San Francisco, USA.

Adelheid Woehrer received her MD in 2006 from the Medical University of Vienna and continued her research there to receive her $\mathrm{PhD}$ in the field of brain tumor epidemiology in Austria. Her major scientific interests are neurooncology, neuroepidemiology, translational research, and biomarker research.

Bernhard Baumann is an associate professor at the Center for Medical Physics and Biomedical Engineering at the Medical University of Vienna. He studied physics at the University of Vienna and received his PhD in medical physics from the Medical University of Vienna in 2009. His research interests are the development of innovative optical methods for biomedical imaging, in particular OCT, and their application for improved diagnostics of diseases in both clinical and preclinical research. 